\title{
Overcoming the quality-quantity tradeoff in dispersion and printing of carbon nanotubes by a repetitive dispersion-extraction process
}

Hiroyuki Shirae $^{a}$, Dong Young Kim ${ }^{a}$, Kei Hasegawa ${ }^{a}$, Taishi Takenobu ${ }^{b}$, Yutaka Ohno ${ }^{c}$, and Suguru Noda ${ }^{a, *}$

${ }^{a}$ Department of Applied Chemistry, School of Advanced Science and Engineering, Waseda University, 3-4-1 Okubo, Shinjuku-ku, Tokyo 169-8555, Japan

${ }^{b}$ Department of Applied Physics, School of Advanced Science and Engineering, Waseda University, 3-4-1 Okubo, Shinjuku-ku, Tokyo 169-8555, Japan

${ }^{c}$ Department of Quantum Engineering, School of Engineering, Nagoya University, Furo-cho, Chikusa-ku, Nagoya 464-8603 Japan

\begin{abstract}
Dispersion-printing processes are essential for the fabrication of various devices using carbon nanotubes (CNTs). Insufficient dispersion results in CNT aggregates, while excessive dispersion results in the shortening of individual CNTs. To overcome this tradeoff, we propose here a repetitive dispersion-extraction process for CNTs. Long-duration ultrasonication (for $100 \mathrm{~min}$ ) produced an aqueous dispersion of CNTs with sodium dodecylbenzene sulfonate with a high yield of $64 \%$, but with short CNT lengths (a few $\mu \mathrm{m}$ ), and poor conductivity in the pinted films $\left(\sim 450 \mathrm{~S} \mathrm{~cm}^{-1}\right)$. Short-duration ultrasonication (for $3 \mathrm{~min}$ ) yielded a CNT dispersion with a very small

\footnotetext{
* Corresponding author: Tel/Fax: +81-3-5286-2769.
}

E-mail address: noda@waseda.jp (S. Noda).
\end{abstract}


yield of $2.4 \%$, but with long CNTs (up to $\sim 20 \mu \mathrm{m}$ ), and improved conductivity in the printed films (2200 S cm$~_{-1}$ ). The remaining sediment was used for the next cycle after the addition of the surfactant solution. $90 \%$ of the CNT aggregates were converted into conductive CNT films within 13 cycles (i.e., within $39 \mathrm{~min}$ ), demonstrating the improved conductivity and reduced energy/time requirements for ultrasonication. CNT lines with conductivities of $1400-2300 \mathrm{~S} \mathrm{~cm}^{-1}$ without doping and sub-100 $\mu \mathrm{m}$ width, and uniform CNT films with $80 \%$ optical transmittance and $50 \Omega /$ sq sheet resistance with nitric acid doping were obtained on polyethylene terephthalate films. 


\section{Introduction}

Carbon nanotubes (CNTs) have a unique one-dimensional nanostructure, and characteristic properties including a high specific surface area, high electrical conductivity and carrier mobility, high tensile strength and mechanical flexibility, and high chemical and thermal stabilities; these properties make them attractive for various applications, including flexible electronics [1-4], and batteries/capacitors [5-8]. Individual CNTs display these highly desirable properties to their maximum extent; in contrast, ensembles of CNTs typically show significantly inferior properties, due to the junctions between the CNTs [9-11]. The dry printing process-in which aerosols of single-wall CNTs (SWCNTs) synthesized via floating-catalyst chemical vapor deposition (CVD) are directly captured by membrane filters, or deposited on plastic films - is a smart approach for the production of high-quality SWCNT films without any damage or contamination [12-15]. Very high performances of 84 and $86 \Omega / \mathrm{sq}$ at $90 \%$ optical transmittance (at $550 \mathrm{~nm}$, excluding the absorption by the base films) were reported for transparent conductive films (TCFs) made using this method after doping with $\mathrm{NO}_{2}$ and $\mathrm{AuCl}_{3}$, respectively $[13,14]$. On the other hand, the wet printing process is advantageous in terms of its scalability and its applicability to CNT aggregates produced using any method [16-21]; however, wet printing methods sometimes result in damage and contamination of the CNTs during the process of dispersing the CNTs in liquids. Chlorosulfonic superacid can be used to dissolve SWCNTs without the application of any external forces to the solution (using methods such as ultrasonication), yielding superior performances of $60 \Omega / \mathrm{sq}$ at $90.9 \%$ optical transmittance (excluding the absorption by the base film); this high performance is due to the absence of any damage resulting from ultrasonication, the absence of any 
surfactant residue in the films, and the high degree of p-type doping by the superacid [16]. However, water-based solution methods are much more popular, because of the safety of the processing and materials used [17-21].

Extensive research efforts have been dedicated to the investigation of milder dispersions of CNTs, and the printing of more conductive CNT films [17-21]. CNTs are often dispersed in water using ultrasonication, and the use of various dispersants, including Triton-X [17,18], sodium dodecyl sulfate (SDS) [19,20], sodium cholate (SC) [20,21], and sodium dodecylbenzene sulfonate (SDBS) [20], has been investigated. Encouraging values have been reported for transparent conductive films (TCFs) made using SWCNTs doped with nitric acid; a sheet resistance of $30 \Omega /$ sq was achieved at $>70 \%$ optical transmittance for SWCNT films prepared on quartz glass substrates using vacuum filtration and transfer [16], and sheet resistances of 70 and $69 \Omega / s q$ were achieved at $80 \%$ optical transmittance for SWCNT films prepared on polyethylene terephthalate (PET) films using spray coating $[19,20]$. However, such films are often made using some fraction of the SWCNTs remaining in the supernatant after the centrifugation of mildly dispersed SWCNTs. Significant amounts of expensive SWCNTs $\left(\sim 1000 \mathrm{USD} \mathrm{g}^{-1}\right)$ are thus wasted; this is a crucial issue that must be solved to achieve practical, inexpensive CNT devices.

In this work, we propose a repetitive dispersion-extraction process to overcome this quality-quantity tradeoff (Fig. 1). CNTs were mildly dispersed and centrifuged, and the supernatant was used to make CNT films. The sediment was then reused (with the addition of a surfactant solution) and mildly dispersed in the next cycle. To examine in detail the effects of changing the dispersion time, the experiments were performed using a batch process. Almost complete conversion ( 90\%) of the as-produced CNT 
aggregates to CNT lines with attractive properties (conductivities of $\sim 2000 \mathrm{~S} \mathrm{~cm}^{-1}$ without doping, and sub-100 $\mu$ m line width) was realized via vacuum filtration of the dispersions on membrane filters with a metal mask [12,13], and subsequent transfer to PET films. Encouraging properties of 105 and $50 \Omega / s q$ (without and with nitric acid doping, respectively) with $80 \%$ optical transmittance (at $550 \mathrm{~nm}$, excluding the absorption by the base film) were realized for the uniform CNT-TCFs on PET films.

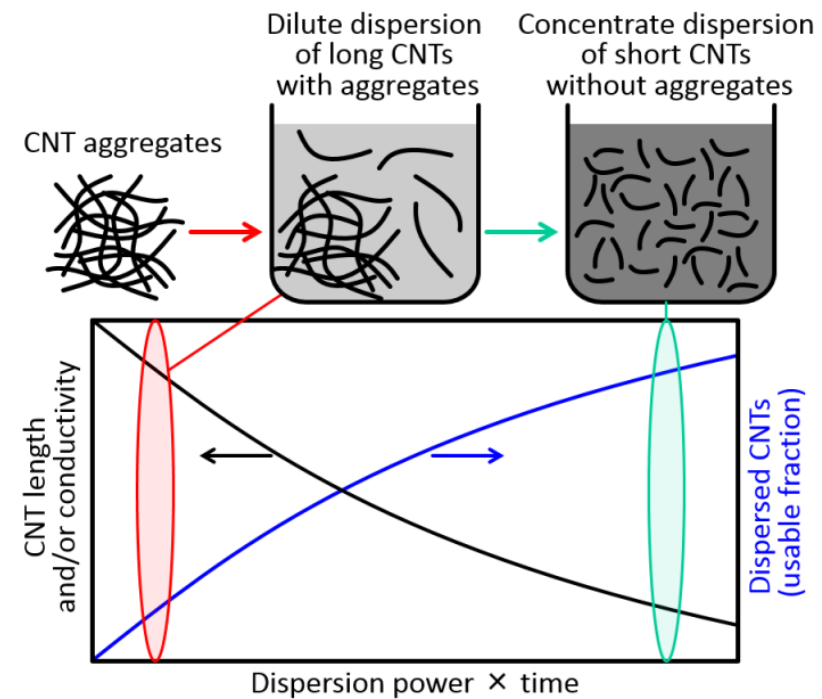

Fig. 1 - Schematic illustrating the quality-quantity tradeoff inherent in the dispersion of CNTs.

\section{Experimental}

2.1. Repetitive process for the dispersion and extraction of CNTs, and evaluation of their dispersion state

The repetitive CNT dispersion-extraction process is illustrated schematically in Fig. 2. As-produced CNTs synthesized via floating-catalyst CVD, the so-called eDIPS method [22] (MEIJO eDIPS, EC grade; Meijo Nano Carbon Co., Ltd., Nagoya, Japan), were used in this work without any purification. The CNTs had a carbon purity of $~ 98 \mathrm{wt} \%$, 
determined by X-ray fluorescence spectroscopy (XRF; Rigaku ZSX Primus 2, Akishima, Japan), and the number of walls and diameter varied from one to three and around 1 to 3 $\mathrm{nm}$, respectively, observed by transmission electron microscope (TEM; JEM-2100F; JEOL, Akishima, Japan) (see Supplementary data, Table S1 and Fig. S1). $100 \mathrm{mg}$ of SDBS (Sigma Aldrich, St. Louis, MO, USA) was dissolved in $20 \mathrm{~mL}$ of deionized water held in a $50 \mathrm{~mL}$ glass bottle. The as-received CNT aggregates were torn into small pieces using tweezers, and $2 \mathrm{mg}$ of the aggregates was added to the bottle (Fig. 2a). The bottle was then placed in a bath-type sonicator (VS-50R, VELVO-CLEAR, Tokyo, Japan) and sonicated at $30 \mathrm{~W}$ and $45 \mathrm{kHz}$ for various times of 3, 10, 30, and $100 \mathrm{~min}$, with the sample solution undergoing convection in the bottle (Fig. 2b). After sonication, the sample in the bottle was centrifuged at $3500 \mathrm{rpm}$ for $20-30 \mathrm{~min}$ (Fig. $2 \mathrm{c}$ ). $15 \mathrm{~mL}$ of the supernatant was used to make the CNT films (Fig. 2d), while $5 \mathrm{~mL}$ of the sediment was used for the next cycle, after the addition of $15 \mathrm{~mL}$ of a $0.5 \mathrm{wt} \%$ aqueous solution of SDBS (Fig. 2a). This cycle was repeated until the CNTs were dispersed almost completely ( $90 \%)$. The concentration of CNTs in the supernatant was determined by measuring the optical absorbance at $550 \mathrm{~nm}$, using ultraviolet-visible spectrophotometry (UV-Vis; V-630, JASCO, Tokyo, Japan), using a calibration curve (Fig. S2). The supernatant was diluted via the addition of $0.5 \mathrm{wt} \%$ SDBS aq. to form a $1 \mathrm{ppmw}$ CNT dispersion, and this dispersion was used to make the films via vacuum filtration.

To investigate the dispersion state of the CNTs, the 1 ppmw CNT dispersion was dip-coated on $\mathrm{Si}$ substrates pretreated using an $\mathrm{H}_{2} \mathrm{SO}_{4}-\mathrm{H}_{2} \mathrm{O}_{2}$ solution. The SDBS adsorbed on the Si substrates was removed from the surface via annealing under a 4 vol\% $\mathrm{H}_{2} / \mathrm{Ar}$ flow, at ambient pressure and $800{ }^{\circ} \mathrm{C}$ for $20 \mathrm{~min}$. The CNTs were then observed using scanning electron microscopy (SEM; Hitachi S-4800, Tokyo, Japan). 
The CNT lines (explained in subsection 2.2) were evaluated using micro-Raman scattering spectroscopy (HR-800, Horiba, Kyoto, Japan), which was performed using an excitation laser wavelength of $488 \mathrm{~nm}$. The ratio of the intensities of the G-band to D-band peaks was used as an index for the CNT quality; this value was 93 for the as-received CNTs.

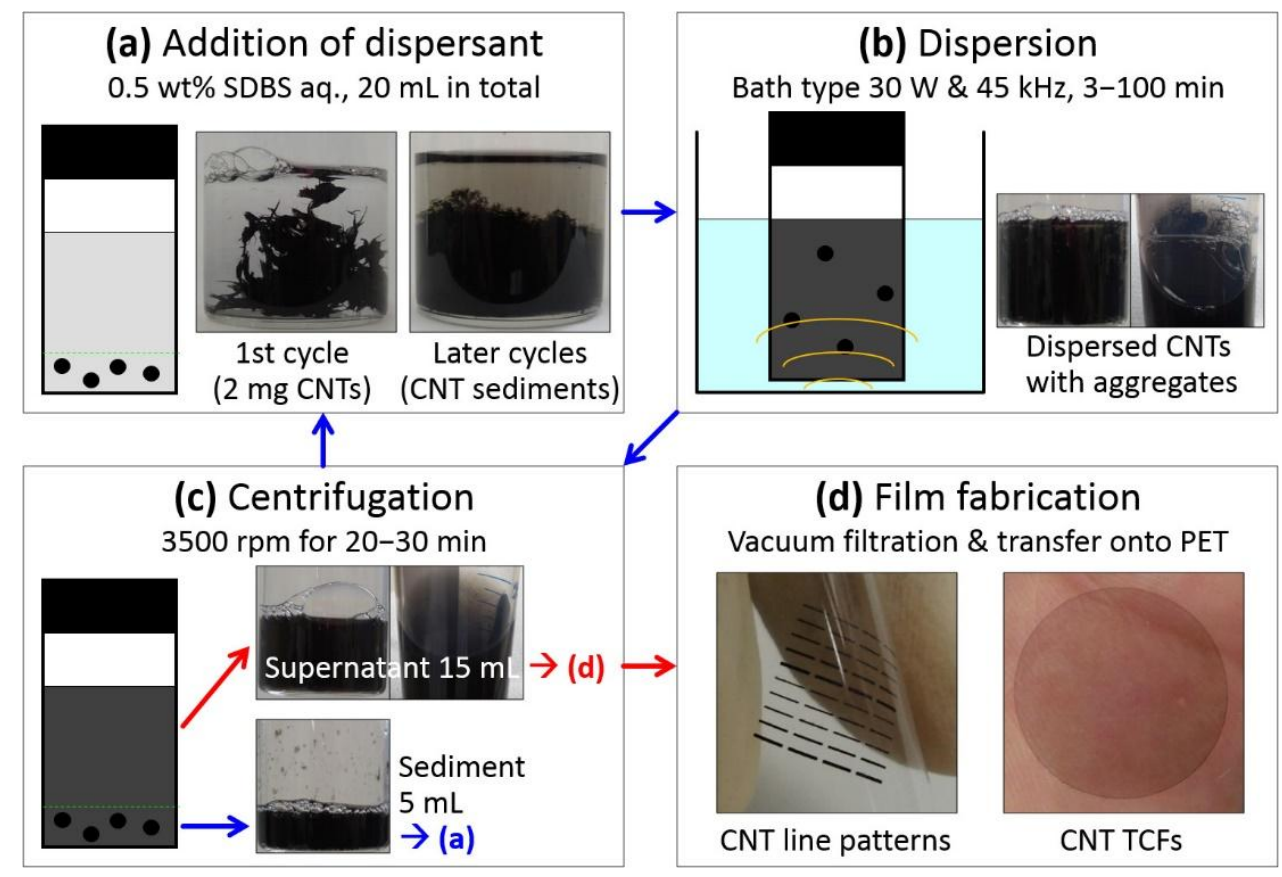

Fig. 2 - The repetitive dispersion-extraction process used to convert the CNT aggregates to CNT films.

\subsection{Fabrication of CNT lines, and evaluation of their structure and conductivity}

To create CNT lines, a metal mask (50 $\mu$ m-thick stainless steel foil with $88-215$ $\mu \mathrm{m}$-wide slits) was used in combination with a polyvinylidene difluoride (PVDF) membrane filter $(0.45 \mu \mathrm{m}$ pore diameter, HVHP, Merck Millipore, Darmstadt, Germany) (Fig. S3a). The CNT dispersion (1 ppmw, $2 \mathrm{~mL}$ ) was vacuum filtrated through the metal mask and membrane filter, and hot deionized water $\left(70{ }^{\circ} \mathrm{C}, 20 \mathrm{~mL}\right)$ 
was then similarly vacuum filtrated to remove the SDBS from the CNT lines. The CNT lines were then transferred from the membrane filter to either Si substrates or PET films (38 $\mu \mathrm{m}$ in thickness, Teijin DuPont Films Japan Limited, Tokyo, Japan) (Fig. S3b). The widths of the CNT lines and the corresponding slits in the metal mask were evaluated using SEM. The thickness of the CNT lines was evaluated using a surface profiler (Alpha-Step 500, KLA Tencor, Milpitas, CA, USA). The conductivity of each CNT line was evaluated by making two-point contact, using Ag paste and a digital multimeter. The distance between the contacts was evaluated using an optical scanner.

2.3. Fabrication of uniform CNT films, and evaluation of their transparency and conduction properties

CNT dispersions prepared using sonication (performed for either 3 or $100 \mathrm{~min}$ ) were used to make uniform CNT films. $30 \mathrm{~mL} \mathrm{CNT} \mathrm{dispersions} \mathrm{of} \mathrm{different} \mathrm{concentrations}$ $(0.033,0.067,0.133$, and 0.27 ppmv) were prepared by diluting 1 ppmv CNT dispersions in $0.5 \mathrm{wt} \%$ SDBS aq. $(1,2,4$, and $8 \mathrm{~mL})$ with deionized water $(29,28,26$, and $22 \mathrm{~mL}$ ). Uniform CNT films (circular shape, $1.8 \mathrm{~cm}$ in diameter, $2.54 \mathrm{~cm}^{2}$ in area) with different CNT loads $(1,2,4,8 \mu \mathrm{g})$ were prepared on a mixed cellulose-ester membrane filter (VCWP, $0.1 \mu \mathrm{m}$ pore diameter, Merck Millipore, Darmstadt, Germany) via vacuum filtration of the dilute dispersions. Immediately after the filtration, and thus before the membrane holding the CNTs had dried, the membrane was dipped in deionized water; this resulted in the CNT film separating from the membrane filter and floating on the surface of the water (Fig. S4). The CNT film was then scooped up using a PET film, and dried on a hot plate at $80^{\circ} \mathrm{C}$ for $5 \mathrm{~min}$. Some CNT-on-PET films were chemically doped by dipping them in concentrated nitric acid for $1 \mathrm{~min}$, followed by 
dipping in deionized water for $5 \mathrm{~s}$, and then drying on a hot plate at $80{ }^{\circ} \mathrm{C}$ for $5 \mathrm{~min}$. The uniform CNT films on the PET films were then evaluated for their transmittance (using UV-Vis spectroscopy performed at $550 \mathrm{~nm}$ ) and sheet resistance (using the four-point-probe method).

\section{Results and discussion}

3.1. Structure and properties of CNT lines prepared from the repetitively dispersed-and-extracted CNT dispersions

Fig. 3 shows typical CNT lines fabricated via the vacuum filtration of the CNT dispersions using the metal mask with a membrane filter, and subsequent transfer onto PET films. Complete transfer to the PET films was possible when few-wall CNTs (FWCNTs) produced by fluidized-bed CVD method, having a rather large diameter of 6-8 nm [23,24], were used (Fig. 3a). Whereas transfer was not complete for the CNT lines made of eDIPS CNTs (Fig. 3b). The eDIPS CNTs with a smaller diameter may have been penetrated deeper into the membrane filter than the FWCNTs during vacuum filtration, causing some difficulty in transfer. The thickness of the CNT lines could be controlled to some extent by changing the amount of filtrated CNTs, but very thick CNT lines $(\gg>1 \mu \mathrm{m})$ could not be made, because they attached to the metal mask. The way for complete transfer of thicker lines of various CNTs is now under study. The eDIPS CNTs were used throughout except for Fig. 3a. The plan-view SEM image in Fig. 3c displayed inter-woven CNT networks with sub-micrometer-scale roughness, possibly reflecting the rough surface of the PVDF membrane filter, which had $0.45 \mu \mathrm{m}$-diameter pores. The CNT lines were transferred from the membrane filter to the PET films by turning the membrane filter upside down; finer membrane filters could be used to obtain 
CNT lines with smoother surfaces. The SEM images showed that the CNT lines were wider than the slits in the metal mask by a few micrometers (Fig. $3 d-m$ ). It is possible that some of the CNTs ended up in the space between the metal mask and the membrane filter. The line width was easily controlled in a linear manner with the slit width down to $85 \mu \mathrm{m}$ (Fig. 3n).
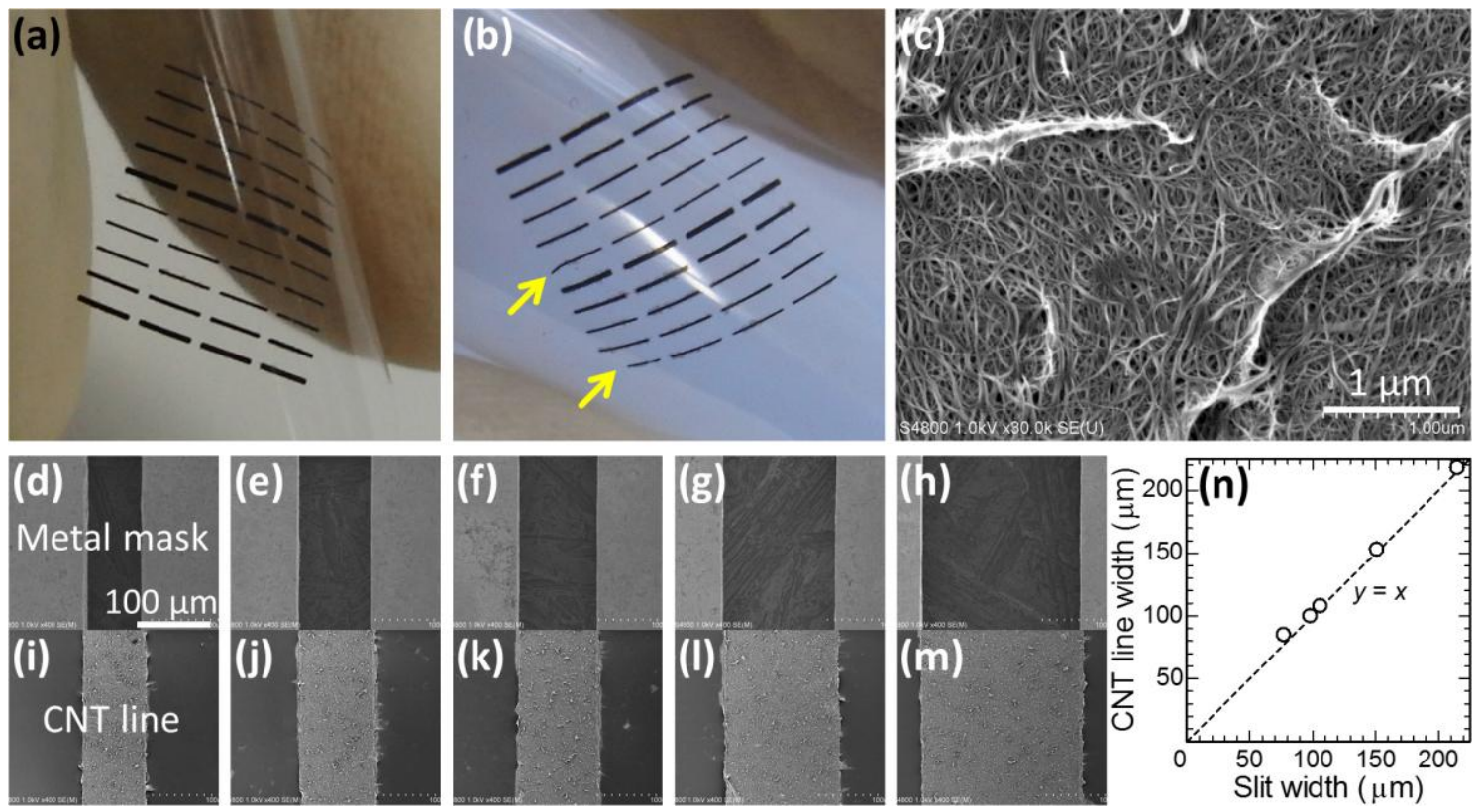

Fig. 3 - Typical CNT lines fabricated via the vacuum filtration of CNT dispersions using the metal mask with a membrane filter, and subsequent transfer onto PET films. Photographs of the CNT lines on a PET film $(a, b)$. Complete transfer was possible for FWCNTs produced by fluidized-bed CVD method [23,24] (a). Some defects, indicated by yellow arrows, were found in the transferred line patterns of the eDIPS CNTs (b). Plan-view SEM image showing the microstructure of a typical CNT line on a $\mathrm{Si}$ substrate (c). SEM images of the slits in the metal mask $(\mathrm{d}-\mathrm{h})$, and corresponding CNT lines on a $\mathrm{Si}$ substrate $(\mathrm{i}-\mathrm{m})$. The scale bar in (d) applies for all images in $(\mathrm{e}-\mathrm{m})$. Relationship between the CNT line width and the slit width (n). 
We then fabricated CNT lines using a series of CNT dispersions prepared using different sonication times and cycle numbers in the repetitive process. Fig. 4a,d shows the dispersion ratio of the CNTs (i.e., the proportion of CNTs extracted to the supernatant from the starting aggregates) plotted against the cycle number and the accumulated sonication time of the repetitive process. A larger number of cycles was required to disperse and extract the CNTs when a shorter sonication time per cycle was used, as expected (Fig. 4a). However, interestingly, the CNTs were dispersed and extracted within a shorter accumulated sonication time when a shorter sonication time per cycle was used (Fig. 4d), meaning that the extraction of the dispersed CNTs promoted the dispersion of the remaining CNT aggregates, thus reducing the time and energy used for sonication. Fig. $4 b$,e shows the ratio of the line length to the resistance plotted against the cycle number and the accumulated sonication time. Because the same amount of CNTs was used to make each CNT line ( $2 \mu \mathrm{g}$ of CNTs for a filtration area of $0.108 \mathrm{~cm}^{2}$ ), these data enabled a direct comparison between the relative conductivity values (i.e., the inverse of the resistance per unit length). Significant changes in the conductivity were observed with changes in the sonication time per cycle; the 3 min-sonication yielded CNT lines that were approximately five times more conductive than those produced using 100 min-sonication. By dividing these relative conductivity values by the cross-sectional area of the lines, we obtained absolute conductivity values (Fig. 4c,f). Fig. S5 shows the height, cross-sectional area, and mass density of the CNT lines. Although the line height values (and thus the cross-sectional area, and the mass density) varied for different cycles, because of the roughness in the CNT lines (Fig. 3c), there was no apparent dependence of the line height on the sonication time per cycle. The variations in height (and thus the cross-sectional area) 
caused variations in the conductivity (Fig. 4c,f), which would have been suppressed by the use of smoother membrane filters with finer pores. It was confirmed that the repetitive dispersion-extraction process, when applied using a shorter sonication time per cycle, was effective in overcoming the quality-quantity tradeoff (Fig. 1); the process using 3 min of sonication per cycle resulted in the almost complete ( 90\%) dispersion and extraction of the mother CNT aggregates after 13 cycles (with an accumulated sonication time of $39 \mathrm{~min})$, producing fairly conductive CNT lines (1400-2300 S cm${ }^{-1}$ without doping).
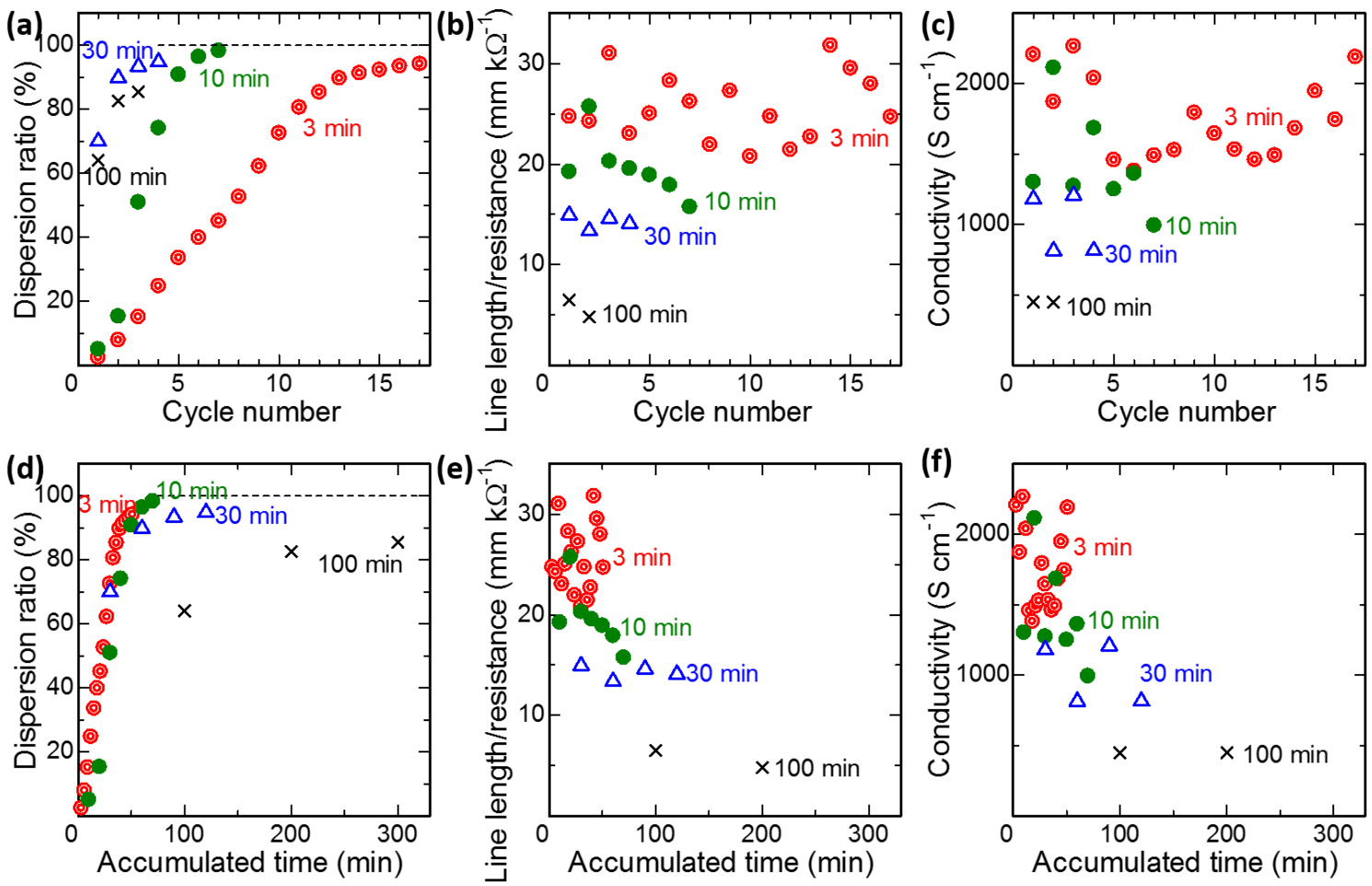

Fig. 4 - Dispersion yields of CNTs (a,d), ratios of line length to resistance for the CNT lines $(\mathrm{b}, \mathrm{e})$, and conductivities of the CNT lines $(\mathrm{c}, \mathrm{f})$ prepared using the repetitive dispersion-extraction process with different sonication times of 3, 10, 30, and $100 \mathrm{~min}$. The data are plotted against the number of cycles used in the repetitive process $(\mathrm{a}-\mathrm{c})$, and the accumulated sonication time $(\mathrm{d}-\mathrm{f})$. The $\mathrm{CNT}$ load was $18.4 \mu \mathrm{g} \mathrm{cm}^{-2}$ for all lines 
$\left(2 \mu \mathrm{g}\right.$ of CNTs for a filtration area of $0.108 \mathrm{~cm}^{-2}$ ). The data shown $(\mathrm{b}, \mathrm{c}, \mathrm{e}, \mathrm{f})$ is for the CNT lines made using the $215 \mu \mathrm{m}$-wide slits.

Compared with the as-received CNT aggregates $\left(\sim 20 \mathrm{~S} \mathrm{~cm}^{-1}\right)$ and the mechanically pressed sheets $\left(\sim 100 \mathrm{~S} \mathrm{~cm}^{-1}\right)$ (Table $\left.\mathrm{S} 2\right)$, the CNT lines prepared through dispersion-filtration showed much higher conductivity of $1000 \mathrm{~S} \mathrm{~cm}^{-1}$ or more. The as-produced CNTs were heavily aggregated and entangled, and thus need be dispersed to form well conductive networks. The CNT lines increased their conductivity upon transferring from the membrane filter to a $\mathrm{SiO}_{2} / \mathrm{Si}$ substrate (Fig. S3), possibly because the CNT lines $(0.5-1 \mu \mathrm{m}$ in thickness) got less wavy and more compact on the flat $\mathrm{Si}$ substrate than the porous membrane filter $(0.45 \mu \mathrm{m}$ in pore size $)$.

We also applied the repetitive dispersion-extraction process for the sub-millimeter-long FWCNTs produced by fluidized-bed CVD method [23,24], however we observed much smaller effect in improving the conductivity (tens of $\%$ ). Such CNTs have a rather large diameter of 6-8 nm and smaller G/D intensity ratios of 1-2 by Raman spectroscopy, and thus are more easily dispersible, resulting in such small effect of the repetitive process. For such long CNTs produced by supported catalysts, shear methods have been reported to be effective [25-27]. Zhang, et al. applied a high-speed stamping shearer with a rotor to centimeter-sized blocks of long CNT arrays and converted them to fluffy CNTs, composed of thin bundles (several $\mu \mathrm{m}$ in diameter) with retained length (several $\mathrm{mm}$ ) [25]. They later dispersed such CNTs in benzyl alcohol and filtrated them to make TCFs with a sheet resistance of $2.5 \mathrm{k} \Omega / \mathrm{sq}$ and a transmittance of $78.6 \%$ [26]. Yoon, et al. applied various dispersion methods to millimeter-long SWCNTs and made composites of $10 \mathrm{wt} \%$ SWCNTs with fluorinated 
rubber [27]. They reported better conductivity for jet mills $\left(28-32 \mathrm{~S} \mathrm{~cm}^{-1}\right)$ than sonicator $\left(20 \mathrm{~S} \mathrm{~cm}^{-1}\right)$ or other methods. But the reported conductivity values in those papers are smaller than this work by two orders of magnitude. Such shearing methods have not been reported to be effective in dispersing highly crystalline, small diameter CNTs possibly because of the too weak shear force in dispersing them although the shear force is mild enough in keeping CNTs long. As explained in Experimental section, we tore CNT aggregates into small pieces using tweezers and then dispersed them by ultrasonication because ultrasonication is not effective for big aggregates. Shearing methods may be effective for pretreatment making such aggregates smaller. We will examine such combination in future.

To examine the change in the CNT length that occurred with increasing sonication time and cycle number, the CNTs were dip-coated on Si substrates, and observed using SEM (Fig. 5). Prior to the SEM observation, the substrates were annealed under a 4 vol\% $\mathrm{H}_{2} / \mathrm{Ar}$ flow, at ambient pressure and $800{ }^{\circ} \mathrm{C}$ for $20 \mathrm{~min}$, which removed SDBS effectively while did not cause any noticeable change in CNT lengths (Fig. S6). 3 min of sonication yielded long CNTs (10-20 $\mu \mathrm{m})$, and short CNTs that were a few $\mu \mathrm{m}$ long (Fig. 5a-c). Similar structure was observed when the number of cycles was increased (Fig. 5a-c), but the fraction of short CNTs (a few $\mu \mathrm{m}$ in length) increased when the sonication time per cycle was increased (Fig. 5d-f). Fig. 5g,f shows the length distributions of these CNTs. For the fixed short sonication time of 3 min per cycle, the fraction of short CNTs increased gradually with increasing cycles (Fig. 5g), whereas for the 1st cycle, the fraction of short CNTs increased remarkably with increasing sonication time per cycle (Fig. 5h). These results agreed with the changes observed in the conductivity of the CNT lines (Fig. 4); CNTs get damaged for longer sonication time 
per cycle. Therefore, it is necessary to take out the CNTs from the sonication bath as soon as they were dispersed, to avoid damage and shortening of the CNTs, and to retain the high conductivity. In contrast, the undispersed CNT aggregates showed less damage, resulting in longer dispersed CNTs for 17 cycles of 3 min-sonication, despite of the longer total sonication time of $51 \mathrm{~min}$, than for the one cycle of 10 and 30-min sonication.
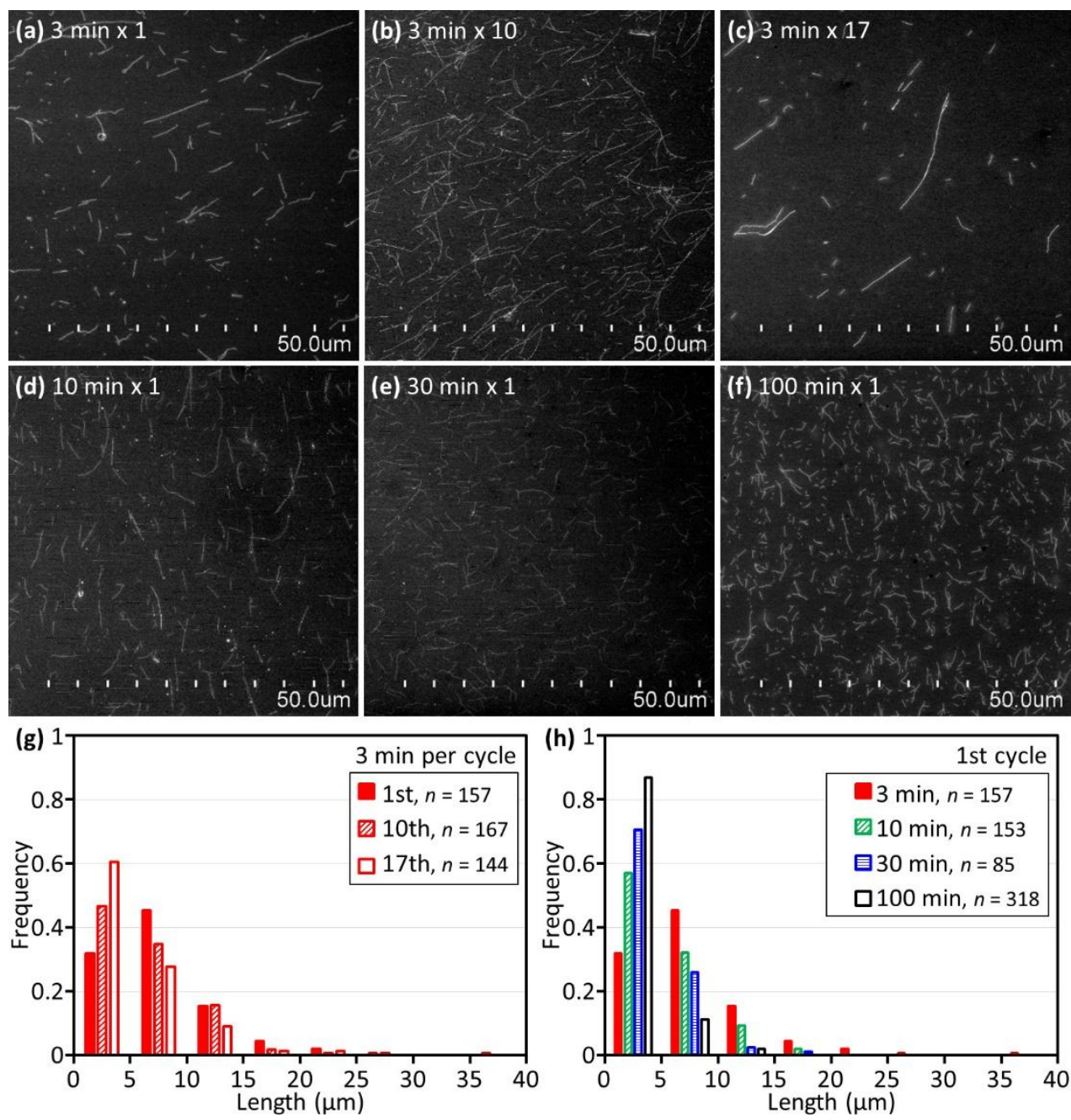

Fig. 5 - $(\mathrm{a}-\mathrm{f})$ SEM images of the CNTs dip-coated on Si substrates; the CNT dispersions were produced using the repetitive dispersion-extraction process, using different sonication times per cycle, and different numbers of cycles. (g,h) Length distributions of the CNTs obtained from the SEM images (a-f). (g) 3-min sonication for 
different cycles and (h) 1st sonication for different sonication times.

The effect of the sonication on the quality of the CNTs was investigated using Raman spectroscopy. Fig. 6a shows Raman spectra for the as-received (and thus as-produced) CNT aggregates and the CNT lines prepared using different CNT dispersions. The spectra were normalized to the G-band intensity, to allow a direct comparison of the D-band intensity for different samples. The as-received CNT aggregates had a very weak D-band peak with a high G/D ratio of 93, illustrating their fairly high quality. After the application of the 3 min-sonication, the D-band peak became visible, but its intensity was unchanged with increases in the cycle number. When the sonication time was increased, the D-band peak became more pronounced; this evidenced the increase in the damage to the CNTs, which consisted of the shortening of individual CNTs and the possible introduction of defects to the CNT sidewalls. The radial breathing mode (RBM) peaks at the low wavenumber region was examined more carefully by taking the spectra at three different point and summing the intensity value for each sample (Fig. 6b). Although a slight shift to higher wavenumber was found from the as-received CNTs to the lines of the 1st cycle of 3-min sonication possibly due to the different aggregation state, no change was observed from the 1st cycle to the 10 th and 17 th cycles. Considering that the dispersions of the 1 st, 10 th, and 17th cycles contained different fractions $(0-2.4,62.2-72.6,93.5-94.2 \mathrm{wt} \%$, respectively) of dispersing CNTs, this result shows that there is no selectivity in CNT diameters for dispersion, at least for SWCNTs. The G/D intensity ratios are plotted against the cycle number in Fig. 6c, and against the accumulated sonication time in Fig. 6d). Even with only 3 min of sonication per cycle, the G/D ratio decreased from 93 (for 
the as-received CNTs) to $\sim 40$, showing that a certain amount of damage had been done to the CNTs. Increases in the sonication time per cycle from 3 to 100 min caused the G/D ratio decrease further, from $\sim 40$ to $<20$. In contrast, for a given sonication time, the $\mathrm{G} / \mathrm{D}$ ratio remained unchanged with increases in the cycle number, and thus the accumulated sonication time. Fig. 6e shows the clear relationship between the conductivity and the G/D ratio for the CNT lines. Both the G/D ratio and conductivity decreased with increasing sonication time per cycle. These results showed that the CNTs were less damaged in their aggregate form, but were damaged once they were dispersed under sonication. These results were in agreement with the SEM observations (Fig. 5); the key to retaining the CNT quality was to take out the CNTs from the sonication bath as soon as they were dispersed. This suggested that milder dispersion conditions might improve the conductivity of the CNT lines; however, simply shortening the sonication time per cycle to $1 \mathrm{~min}$ was not effective. It is likely that other measures-such as reducing the sonication power, and/or using different surfactants—will be required. 
(a)
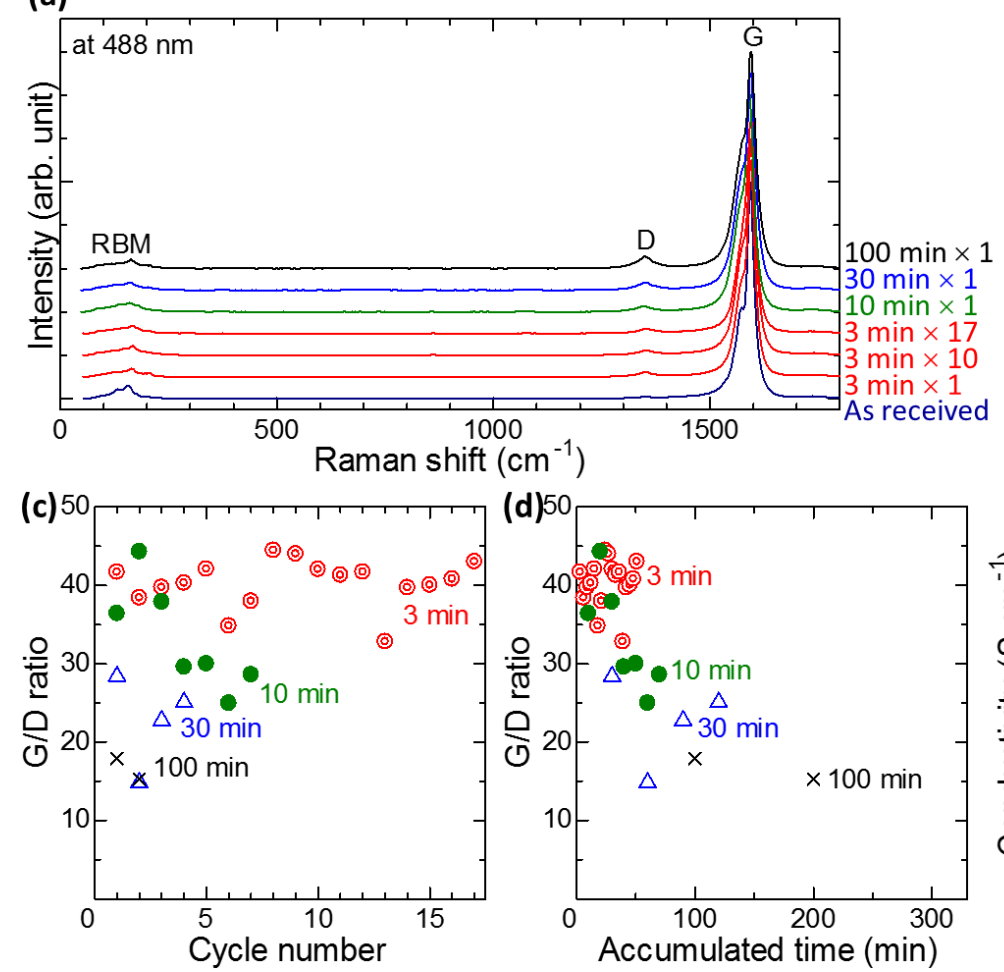

(b) Diameter (nm)
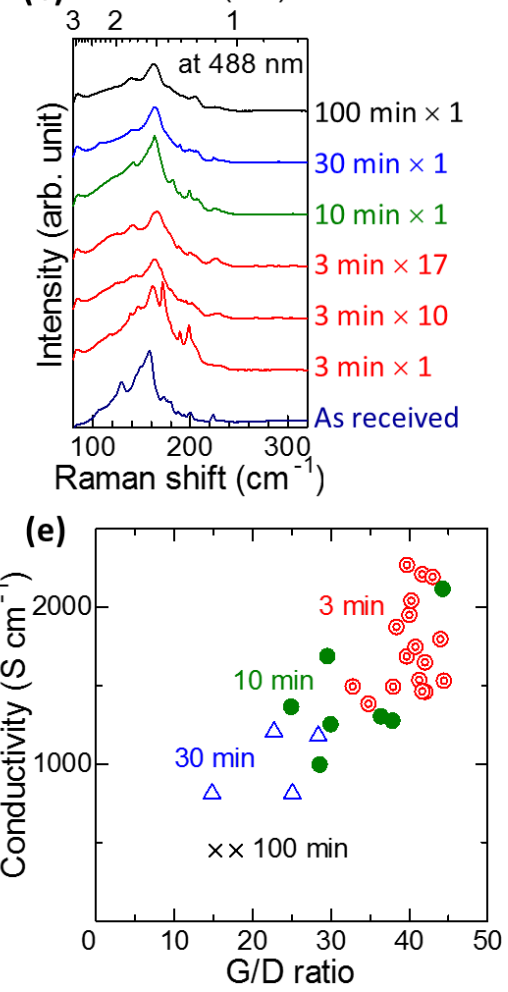

Fig. 6 - (a) Raman spectra for the as-received CNT aggregates, and the CNT lines prepared from the dispersions using the repetitive dispersion-extraction process with different sonication times and cycle numbers. The intensity was normalized to the G-band peak intensity. (b) Raman spectra for the low wavenumber region. Diameter of CNTs calculated using the relationship between diameter $d(\mathrm{~nm})$ and Raman shift $\omega$ $\left(\mathrm{cm}^{-1}\right), d=248 / \omega[28]$, is also shown. (c) Change in the G/D ratio with changes in the cycle number. (d) Change in the G/D ratio with changes in the accumulated sonication time. (e) Relationship between the conductivity and the G/D ratio for the CNT lines.

Fig. 7 summarizes the quality-quantity tradeoff in a one-batch dispersion. A short sonication time of 3 min produced a dispersion containing long CNTs, which yielded high-quality CNT lines (conductivity of $2200 \mathrm{~S} \mathrm{~cm}^{-1}$ and G/D ratio of 42) but a very small dispersion yield of $2.4 \%$. A long sonication time of $100 \mathrm{~min}$ produced a 
dispersion containing short CNTs, which yielded low-quality CNT lines (conductivity of $450 \mathrm{~S} \mathrm{~cm}^{-1}$ and $\mathrm{G} / \mathrm{D}$ ratio of 18 ) but a high dispersion yield of $64 \%$. The repetitive dispersion-extraction process overcame this tradeoff; 13 cycles of 3-min sonication yielded a high-quality CNT dispersion with a high dispersion yield of $\sim 90 \%$. The development of a continuous process based on simultaneous dispersion-extraction with a short residence time for the solution in the sonication bath is now underway. 


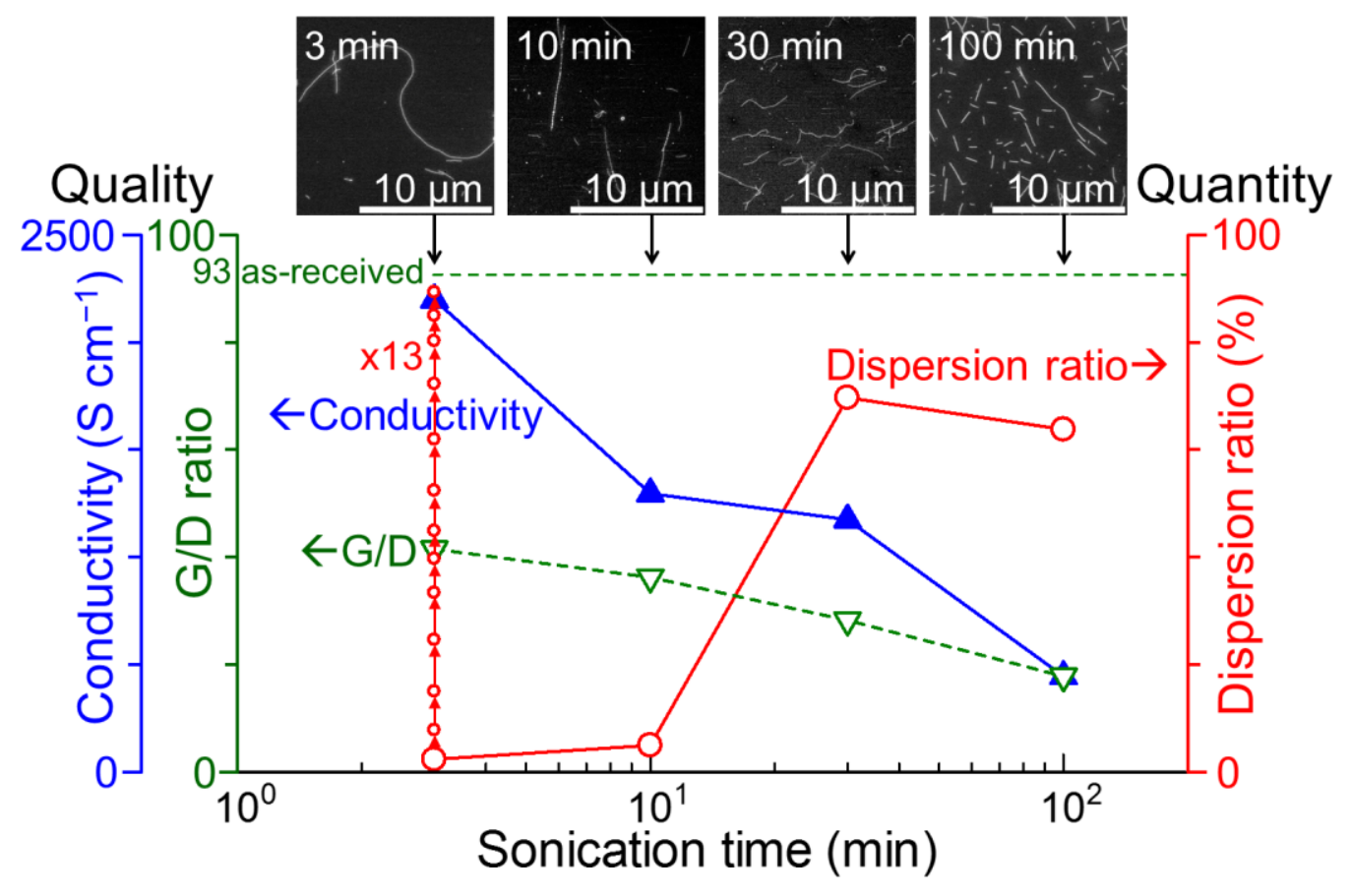

Fig. 7 - Summary of the quality-quantity tradeoff in a one-batch dispersion of CNTs. The data were extracted for the first cycle from Figs. 4-6. The vertical red arrows show the change in the accumulated dispersion ratio with increasing cycles for 3-min sonication. The repetitive dispersion-extraction process, when used with a short sonication time per cycle $(3 \min \times 13)$ overcame this quality-quantity relationship, enabling a high-quality dispersion to be achieved with a dispersion yield of $\sim 90 \%$.

\subsection{Transparency and conduction properties of CNT-TCFs}

The repetitive dispersion-extraction process yielded CNT dispersions of similar quality for different numbers of cycles, unless the sonication time per cycle was changed. We therefore prepared one-batch dispersions of CNTs using sonication times of 3 and 100 min, and used these dispersions to make CNT-TCFs. Fig. 8 illustrates the transparency and conduction properties of the CNT-TCFs made on PET films. For a uniform material with a constant optical absorption coefficient $a$ and an electric resistivity $\rho$, it is possible 
to derive simple relationships between the optical transmittance $T$ and the sheet resistance $R_{\mathrm{S}}$ :

$$
\begin{aligned}
& T=\exp (-a t) \\
& R_{\mathrm{S}}=\rho / t \\
& T=\exp \left(-a \rho / R_{\mathrm{S}}\right)
\end{aligned}
$$

where $t$ is the film thickness. In the case of CNT films, however, the mass density changes depending on the dispersion and printing conditions, and it is therefore not appropriate to substitute CNT film thickness values for $t$ in Eqs. (1) and (2). Fig. 8a shows the relationships between the optical transmittance and the areal density of the CNT films. Interestingly, all of the data could be plotted on a single curve; this indicated that the transmittance was governed by the areal CNT density, and was little affected by the CNT length or the CNT doping, at least for the CNTs used in this work, which had distributions in diameter and number of walls (Fig. S1). The areal densities were 0.33, 0.67 , and $1.42 \mu \mathrm{g} \mathrm{cm}^{-2}$ for optical transmittances of 95,90 , and $80 \%$, respectively. These values could be useful for obtaining a rough estimate of the amount of CNTs required to make CNT-TCFs. Fig. $8 b$ shows the relationship between the optical transmittance and the sheet resistance. Theoretical curves for Eq. (3) are also shown in the figure for different $a \rho$ values. This plot clearly showed that the TCFs produced using mildly dispersed CNTs (3 min of sonication) showed superior performance. Encouraging values of $105 \Omega / \mathrm{sq}$ at $79.6 \%$ and $50 \Omega / \mathrm{sq}$ at $80.0 \%$ were observed for the non-doped and doped CNT films, respectively. The stability of the resistance of the doped CNT-TCFs was examined in a laboratory environment (Fig. S7). The resistance gradually increased, and then stabilized at $130-140 \%$ of the initial resistance after $200 \mathrm{~h}$. The repetitive dispersion-extraction process enabled the conversion of $90 \%$ of the 
as-produced CNT aggregates into high-quality CNT-TCFs.
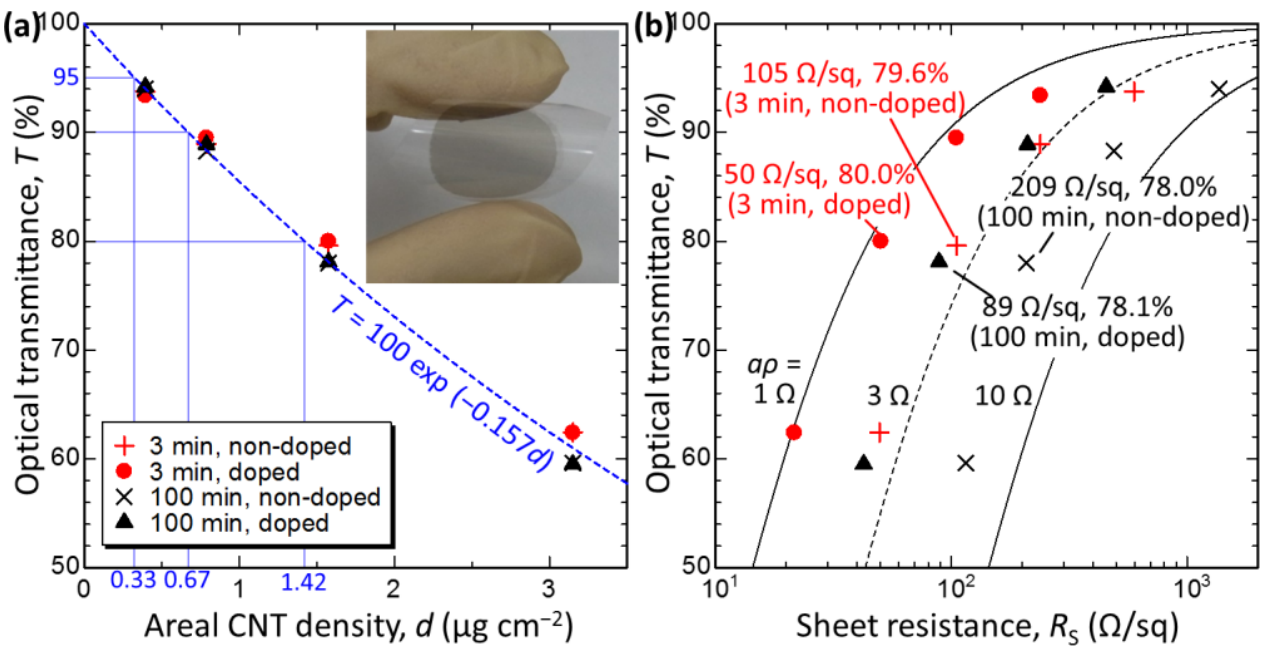

Fig. 8 - Transparency and conduction properties of the CNT-TCFs produced on PET films using CNT dispersions prepared via one-batch sonication for 3 or $100 \mathrm{~min}$. Some of the CNT-TCFs were doped by dipping in nitric acid. (a) Relationship between the optical transmittance at $550 \mathrm{~nm}$ (excluding the absorption of the base PET film) and the areal CNT density. The inset is a photograph showing a typical flexible TCF with a transmittance of $\sim 80 \%$. (b) Relationship between the optical transmittance and sheet resistance. Theoretical curves for Eq. (3) are also shown for different $a \rho$ values of 1, 3, and $10 \Omega$.

The TCF performance is often analyzed using the direct current and optical conductivities, $\sigma_{\mathrm{dc}}$ and $\sigma_{\mathrm{op}}$, respectively, using a relationship:

$$
T=\left(1+\frac{1}{2 R_{\mathrm{S}}} \sqrt{\frac{\mu_{0}}{\varepsilon_{0}}} \frac{\sigma_{\mathrm{op}}}{\sigma_{\mathrm{dc}}}\right)^{-2}
$$

where $\mu_{0}=4 \pi \times 10^{-7} \mathrm{~N} \mathrm{~A}^{-2}$ and $\varepsilon_{0}=8.854 \times 10^{-12} \mathrm{C}^{2} \mathrm{~N}^{-1} \mathrm{~m}^{-2}$ are the free space permeability and permittivity, respectively [29]. Eq. (4) also fits the results well expect for the films of high transmittance of $\sim 95 \%$ (Fig. S8). The $\sigma_{\mathrm{dc}} / \sigma_{\mathrm{op}}$ ratio is an important 
factor showing the TCF performance, and was 32.0 for the doped CNT-TCFs made from the CNT dispersion by 3-min sonication. This value is encouraging; higher than the value of 25.3 in the previous report [30].

In this work, we used a bath-type sonicator with a fixed power of $30 \mathrm{~W}$ and frequency of $45 \mathrm{kHz}$ and reduced the sonication time to make dispersion milder. Changing the sonication power or frequency would also be effective to realize mild dispersion. We used a tip-type sonicator (Hielscher UP400S, Teltow, Germany; maximum power of $400 \mathrm{~W}$, fixed frequency of $24 \mathrm{kHz}$ ) and examined the effect of sonication power (Fig. S9). The concentration of dispersed CNTs were different for two different runs (Fig. S9a), possibly due to the position of the CNT aggregates relative to the sonication tip. We prepared CNT-TCFs and evaluated their performance and obtained $\sigma_{\mathrm{dd}} / \sigma_{\mathrm{op}}$ values (Fig. S9b). These values had a good correlation with the dissolved CNT concentrations; the value decreased with the increasing concentrations of dissolved CNTs (Fig. S9c). This correlation shows that CNTs are dispersed and damaged more as the sonication energy (power and time) increases. We will work further on pretreatments of the CNT aggregates (such by shearing methods), optimization of sonication time, power, and frequency, and continuous operation for repetitive dispersion-extraction, toward the practical dispersion process for loss-free, high quality CNT dispersions.

\section{Conclusions}

To overcome the quality-quantity tradeoff in dispersing and printing CNTs, we proposed and examined a repetitive dispersion-extraction process. As the sonication time in SDBS aq. was increased from 3 to $100 \mathrm{~min}$, the dispersion ratio increased from $2.4 \%$ to 
$64 \%$, while the quality of the CNTs degraded; the proportion of $10-20 \mu \mathrm{m}$-long CNTs decreased and the proportion of CNTs with lengths of a few $\mu \mathrm{m}$ increased, the G/D ratio decreased from 42 to 18 , and the conductivity decreased from 2200 to $450 \mathrm{~S} \mathrm{~cm}^{-1}$. The repetitive cycle consisting of $3 \mathrm{~min}$ of sonication, centrifugation and extraction of the supernatant, and re-dispersion of the sediment overcame this tradeoff; the CNTs were almost completely dispersed $(\sim 90 \%)$ within 13 cycles, and the high quality of the dispersed CNTs was retained. The extraction of the dispersed CNTs from the solution not only prevented damage to the CNTs, but also promoted the dispersion of the CNT aggregates; $90 \%$ of CNTs were dispersed after an accumulated sonication time of 39 min (3 min of sonication per cycle over 13 cycles). The fact that the quality of the dispersed CNTs was maintained in the later cycles showed that less damage was inflicted on the CNTs when they were in their aggregate form.

Vacuum filtration on a membrane filter with a metal mask enabled the loss-free printing of CNT line patterns with sub-100 $\mu \mathrm{m}$ width on PET films although further investigation is needed for defect-free transfer. CNT-TCFs were prepared on PET films using the CNT dispersions produced using 3 min-sonication. Encouraging properties of 105 and $50 \Omega /$ sq (without and with nitric acid doping, respectively) with $80 \%$ optical transmittance were realized. The repetitive process enabled the conversion of $90 \%$ of the as-produced CNT aggregates into high-quality CNT films; the batch operation used in this work was, however, time-consuming. The development of a continuous process based on simultaneous dispersion-extraction with a short residence time for the solution in the sonication bath is now underway, with the aim of moving toward inexpensive printed CNT films and devices. 


\section{Acknowledgements}

The authors thank Meijo Nano Carbon Co., Ltd. for kindly providing us with their MEIJO eDIPS CNTs, Mr. Shinpei Enomoto at Kagami Memorial Research Institute for Materials Science and Technology, Waseda University for TEM observation, and Mr. Quintero Ricardo at Waseda University for XRF measurement. The authors also thank Dr. Kazunori Yamamoto, Mr. Eisuke Haba, and Mr. Shunsuke Ueda at Hitachi Chemical Company, Ltd. for their collaboration at the very early stage of this work. This work is financially supported by the Advanced Low Carbon Technology Research and Development Program (ALCA) by JST, Japan. H.S. acknowledges the Leading Graduate Program in Science and Engineering, Waseda University from MEXT, Japan. 


\section{References}

[1] Cao Q, Kim HS, Pimparkar N, Kulkarni JP, Wang CJ, Shim M, et al. Medium-scale carbon nanotube thin-film integrated circuits on flexible plastic substrates. Nature 2008;454(7203):495-500.

[2] Takenobu T, Takahashi T, Kanbara T, Tsukagoshi K, Aoyagi Y, Iwasa Y. High-performance transparent flexible transistors using carbon nanotube films. Appl Phys Lett 2006;88(3):033511.

[3] Sun DM, Timmermans MY, Tian Y, Nasibulin AG, Kauppinen EI, Kishimoto S, et al. Flexible high-performance carbon nanotube integrated circuits. Nat Nanotechnol 2011;6(3):156-61.

[4] Sun DM, Timmermans MY, Kaskela A, Nasibulin AG, Kishimoto S, Mizutani T, et al. Mouldable all-carbon integrated cirucits. Nat Commun 2013;4:2302.

[5] Futaba DN, Hata K, Yamada T, Hiraoka T, Hayamizu Y, Kakudate Y, et al. Shape-engineerable and highly densely packed single-walled carbon nanotubes and their application as super-capacitor electrodes. Nat Mater 2006;5(12):987-94.

[6] Cui LF, Hu L, Choi JW, Cui Y. Light-weight free-standing carbon nanotube-silicon films for anodes of lithium ion batteries. ACS Nano 2010;4(7):3671-8.

[7] Lee SW, Gallant BM, Lee Y, Yoshida N, Kim DY, Yamada Y, et al. Self-standing positive electrodes of oxidized few-walled carbon nanotubes for light-weight and high-power lithium batteries. Energy Environ Sci 2012;5(1):5437-44.

[8] Quintero R, Kim DY, Hasegawa K, Yamada Y, Yamada A, Noda S. Carbon nanotube 3D current collectors for lightweight, high performance and low cost supercapacitor electrodes. RSC Adv 2014;4(16):8230-7.

[9] Stadermann M, Papadakis SJ, Falvo MR, Novak J, Snow E, Fu Q, et al. Nanoscale 
study of conduction through carbon nanotube networks. Phys Rev B 2004;69(20):201402.

[10] Nirmalraj PN, Lyons PE, De S, Coleman JN, Boland JJ. Electrical connectivity in single-walled carbon nanotube networks. Nano Lett 2009;9(11):3890-5.

[11] Znidarsic A, Kaskela A, Laiho P, Gaberscek M, Ohno Y, Nasibulin AG, et al. Spatially resolved transport properties of pristine and doped single-walled carbon nanotube networks. J Phys Chem C 2013;117(25):13324-30.

[12]Kaskela A, Nasibulin AG, Timmermans MY, Aitchison B, Papadimitratos A, Tian Y, et al. Aerosol-synthesized SWCNT networks with tunable conductivity and transparency by a dry transfer technique. Nano Lett 2010;10(11):4349-55.

[13] Nasibulin AG, Kaskela A, Mustonen K, Anisimov AS, Ruiz V, Kivisto S, et al. Multifunctional free-standing single-walled carbon nanotube films. ACS Nano 2011;5(4):3214-21.

[14] Reynaud O, Nasibulin AG, Anisimov AS, Anoshkin IV, Jiang H, Kauppinen EI. Aerosol feeding of catalyst precursor for CNT synthesis and highly conductive and transparent film fabrication. Chem Eng J 2014;255:134-40.

[15] Fukaya N, Kim DY, Kishimoto S, Noda S, Ohno Y. One-step sub-10 $\mu$ m patterning of carbon-nanotube thin films for transparent conductor applications. ACS Nano 2014;8(4):360-4.

[16] Hecht DS, Heintz AM, Lee R, Hu L, Moore B, Cucksey C, et al. High conductivity transparent carbon nanotube films deposited from superacid. Nanotechnology 2011;21(7):075201.

[17] Saran N, Parikh K, Suh DS, Muoz E, Kolla H, Manohar SK. Fabrication and characterization of thin films of single-walled carbon nanotube bundles on flexible 
plastic substrates. J Am Chem Soc 2004;126(14):4462-3.

[18] Wu ZC, Chen Z, Du X, Logan JM, Sippel J, Nikolou M, et al. Transparent, conductive carbon nanotube films. Science 2004;305(5688):1273-6.

[19] Geng HZ, Kim KK, So KP, Lee YS, Chang Y, Lee YH. Effect of acid treatment on carbon nanotube-based flexible transparent conducting films. J Am Chem Soc 2007;129(25):7758-9.

[20] Goak JC, Lee SH, Han JH, Jang SH, Kim KB, Seo Y, et al. Spectroscopic studies and electrical properties of transparent conductive films fabricated by using surfactant-stabilized single-walled carbon nanotube suspensions. Carbon 2011;49(13):4301-13.

[21] Miyata Y, Yanagi K, Maniwa Y, Kataura H. Highly stabilized conductivity of metallic single wall carbon nanotube thin films. J Phys Chem C 2008;112(10):3591-6.

[22] Saito T, Xu W-C, Ohshima S, Ago H, Yumura M, Iijima S. Supramolecular catalysts for the gas-phase synthesis of single-walled carbon nanotubes. J Phys Chem B 2006;110(12):5849-53.

[23] Kim DY, Sugime H, Hasegawa K, Osawa T, Noda S. Sub-millimeter-long carbon nanotubes repeatedly grown on and separated from ceramic beads in a single fluidized bed reactor. Carbon 2011;49(6):1972-9.

[24] Chen Z, Kim DY, Hasegawa K, Osawa T, Noda S. Over 99.6 wt\%-pure, sub-millimeter-long carbon nanotubes realized by fluidized-bed with careful control of the catalyst and carbon feeds. Carbon 2014;80:339-50.

[25] Zhang Q, Xu G-H, Huang J-Q, Zhou W-P, Zhao M-Q, Wang Y, et al. Fluffy carbon nanotubes produced by shearing vertically aligned carbon nanotube arrays. 
Carbon 2009;47(2):544-7.

[26] Xu G-H, Zhang Q, Huang J-Q, Zhao M-Q, Zhou W-P, Wei F. A two-step shearing strategy to disperse long carbon nanotubes from vertically aligned multiwalled carbon nanotube arrays for transparent conductive films. Langmuir 2010;26(4):2798-804.

[27] Yoon H, Yamashita M, Ata S, Futaba DN, Yamada T, Hata K. Controlling exfoliation in order to minimize damage during dispersion of long SWCNTs for advanced composites. Sci Rep 2014;4:3907.

[28] Jorio A, Saito R, Hafner JH, Lieber CM, Hunter M, McClure T, et al. Trigonal warping effect of carbon nanotubes. Phys Rev B 2000;61(4):2981-90.

[29] Hecht D, Hu L, Grüner G. Conductivity scaling with bundle length and diameter in single walled carbon nanotube networks. App Phys Lett 2006:89(13);133112.

[30] Geng H-Z, Lee D S, Kim K K, Han G H, Park H K, Lee Y H. Absorption spectroscopy of surfactant-dispersed carbon nanotube film: Modulation of electronic structures. Chem Phys Lett 2008;455(4-6):275-8. 


\section{Figure captions}

Fig. 1 - Schematic illustrating the quality-quantity tradeoff inherent in the dispersion of CNTs.

Fig. 2 - The repetitive dispersion-extraction process used to convert the CNT aggregates to CNT films.

Fig. 3 - Typical CNT lines fabricated via the vacuum filtration of CNT dispersions using the metal mask with a membrane filter, and subsequent transfer onto PET films. Photographs of the CNT lines on a PET film (a,b). Complete transfer was possible for FWCNTs produced by fluidized-bed CVD method [23,24] (a). Some defects, indicated by yellow arrows, were found in the transferred line patterns of the eDIPS CNTs (b). Plan-view SEM image showing the microstructure of a typical CNT line on a Si substrate (c). SEM images of the slits in the metal mask $(d-h)$, and corresponding CNT lines on a Si substrate $(i-m)$. The scale bar in $(d)$ applies for all images in $\left(\mathrm{e}^{-}-\mathrm{m}\right)$. Relationship between the CNT line width and the slit width (n).

Fig. 4 - Dispersion yields of CNTs (a,d), ratios of line length to resistance for the CNT lines $(b, e)$, and conductivities of the CNT lines (c,f) prepared using the repetitive dispersion-extraction process with different sonication times of 3, 10, 30, and 100 min. The data are plotted against the number of cycles used in the repetitive process $(a-c)$, and the accumulated sonication time $(d-f)$. The CNT load was 18.4 $\mu \mathrm{g} \mathrm{cm}^{-2}$ for all lines $\left(2 \mu \mathrm{g}\right.$ of CNTs for a filtration area of $\left.0.108 \mathrm{~cm}^{-2}\right)$. The data shown (b,c,e,f) is for the CNT lines made using the $215 \mu \mathrm{m}$-wide slits. 
Fig. 5 - $(\mathrm{a}-\mathrm{f}) \mathrm{SEM}$ images of the CNTs dip-coated on Si substrates; the CNT dispersions were produced using the repetitive dispersion-extraction process, using different sonication times per cycle, and different numbers of cycles. (g,h) Length distributions of the CNTs obtained from the SEM images $(a-f)$. (g) 3-min sonication for different cycles and (h) 1st sonication for different sonication times.

Fig. 6 - (a) Raman spectra for the as-received CNT aggregates, and the CNT lines prepared from the dispersions using the repetitive dispersion-extraction process with different sonication times and cycle numbers. The intensity was normalized to the G-band peak intensity. (b) Raman spectra for the low wavenumber region. Diameter of CNTs calculated using the relationship between diameter $d(\mathrm{~nm})$ and Raman shift $\omega\left(\mathrm{cm}^{-1}\right), d=248 / \omega$ [28], is also shown. (c) Change in the G/D ratio with changes in the cycle number. (d) Change in the G/D ratio with changes in the accumulated sonication time. (e) Relationship between the conductivity and the $\mathrm{G} / \mathrm{D}$ ratio for the CNT lines.

Fig. 7 - Summary of the quality-quantity tradeoff in a one-batch dispersion of CNTs. The data were extracted for the first cycle from Figs. 4-6. The vertical red arrows show the change in the accumulated dispersion ratio with increasing cycles for 3-min sonication. The repetitive dispersion-extraction process, when used with a short sonication time per cycle (3 $\min \times 13)$ overcame this quality-quantity relationship, enabling a high-quality dispersion to be achieved with a dispersion yield of $\sim 90 \%$.

Fig. 8 - Transparency and conduction properties of the CNT-TCFs produced on PET films using CNT dispersions prepared via one-batch sonication for 3 or $100 \mathrm{~min}$. 
Some of the CNT-TCFs were doped by dipping in nitric acid. (a) Relationship between the optical transmittance at $550 \mathrm{~nm}$ (excluding the absorption of the base PET film) and the areal CNT density. The inset is a photograph showing a typical flexible TCF with a transmittance of $\sim 80 \%$. (b) Relationship between the optical transmittance and sheet resistance. Theoretical curves for Eq. (3) are also shown for different $a \rho$ values of 1,3 , and $10 \Omega$. 